\title{
Preventing transfusion-associated graft-versus-host disease: state of the art
}

This article was published in the following Dove Press journal:

International Journal of Clinical Transfusion Medicine

7 January 2015

Number of times this article has been viewed

\section{Loren D Fast}

Division of Hematology/Oncology, Rhode Island Hospital and Warren Alpert School of Medicine at Brown University, Providence, RI, USA
Correspondence: Loren D Fast Division of Hematology/Oncology, Rhode Island Hospital, I Hoppin Street, Coro West Suite 5.0.I, Providence, RI, USA

$\mathrm{Tel}+\mathrm{I} 40$ I 444809 I

Fax + I 40I 444466 I

Email loren_fast@brown.edu
Abstract: The transfer of pathogens and the induction of immune responses are deleterious consequences that can result from the transfusion of blood products. Transfusion-associated graft-versus-host disease (TA-GVHD), the most severe immune consequence, occurs when recipient immune responses are incapable of effectively eliminating donor leukocytes, permitting unabated responses of the donor T lymphocytes. Currently, prevention of TA-GVHD is routinely accomplished by exposing blood products to $\gamma$-irradiation in order to prevent donor $\mathrm{T}$ cell proliferation. Alternative protocols are being developed to meet the challenges associated with the use of $\gamma$-irradiation. Use of pathogen reduction protocols, which interfere with nucleic acid replication by modifying nucleic acids, are increasing. Comparison of pathogen reduction protocols with $\gamma$-irradiation have found that both protocols are equally effective in preventing $\mathrm{T}$ lymphocyte proliferation and GVHD responses when testing in both in vitro and in vivo models. The potential use of pathogen reduction protocols to treat whole blood prior to separation into its components could provide a cost-effective method for preventing TA-GVHD in the future.

Keywords: blood transfusion, GVHD, pathogen reduction, irradiation

\section{Introduction}

Restoration of diminished blood cell levels by the transfusion of blood products can provide significant benefit to the recipient. However, transfusions can also introduce infectious pathogens and trigger immune responses. These immune responses can include donor anti-recipient immune responses as well as recipient anti-donor immune responses induced by the donor leukocytes acting as antigen-presenting cells or as a source of antigen. While recipient anti-donor responses are usually able to eliminate donor leukocytes, settings in which the recipient anti-donor responses are impaired permits unabated donor anti-recipient responses, resulting in transfusion-associated graft-versus-host disease (TA-GVHD), a response that is almost uniformly fatal. ${ }^{1}$

TA-GVHD typically presents within 2-30 days following transfusion with an erythematous, maculopapular rash, fever, elevated liver enzymes, often with associated hepatomegaly and jaundice, plus gastrointestinal symptoms, including nausea, vomiting, and diarrhea. ${ }^{1,2}$ TA-GVHD is often confused with allergic reactions to drugs or viral infections. In TA-GVHD, the donor anti-recipient responses cause marked bone marrow aplasia. The resulting neutropenia renders the patient highly susceptible to infection. The bone marrow aplasia presents a difficult hurdle to overcome when attempting to treat the patient with ongoing TA-GVHD. As a result, emphasis has been placed on preventing the development of TA-GVHD. ${ }^{1,2}$ 


\section{Risk factors for TA-GVHD}

The generation of TA-GVHD requires prolonged donor cell persistence. Studies tracking the fate of transfused donor white blood cells (WBCs) have found that normally $>99.9 \%$ of the donor WBCs have been eliminated from the blood by 3 days after transfusion. ${ }^{3}$ Studies in the mouse model have shown that recipient cluster of differentiation $8(C D 8)+$ cells are primarily responsible for the elimination of large numbers of allogeneic donor cells and that alloantibody production is responsible for the elimination of allogeneic cells in the absence of CD8+ cells (Figure 1). ${ }^{4,5}$ Both of these responses are facilitated by the presence of activated donor $\mathrm{CD} 4+$ cells. $^{5}$ Other possible recipient mechanisms responsible for the elimination or inhibition of donor cells could include natural killer (NK) cells and dendritic cells. ${ }^{6-12}$ These elimination responses are quite potent, as infusion of an unfractionated pheresis product containing $2 \times 10^{10} \mathrm{CD} 3+$ cells into nonconditioned, haploidentical, refractory leukemia patients enrolled in a clinical trial resulted in almost complete elimination of circulating donor cells in the blood by day $7 .{ }^{13}$ TA-GVHD would be predicted to occur when these elimination responses are compromised, thereby permitting prolonged survival of donor cells with the ability to proliferate and to generate donor anti-recipient responses.

A transfusion from a human leukocyte antigen (HLA) homozygous donor into a recipient who shares one haplotype with the donor would be a scenario in which the recipient anti-donor elimination responses are compromised. ${ }^{14}$ This is because the recipient immune cells, especially alloreactive

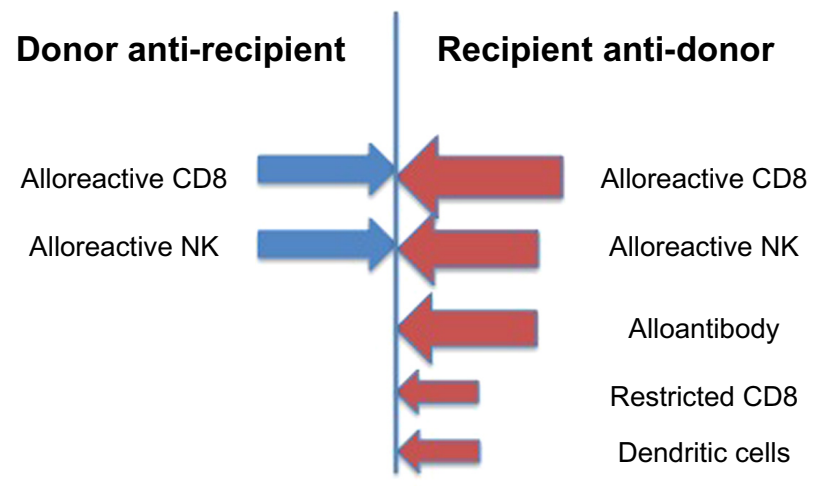

Figure I Transfusion-induced immune responses.

Notes: The recipient anti-donor and donor anti-recipient immune responses potentially occurring following the transfusion of blood products are illustrated. The consequence of usually having many more recipient lymphocytes than donor lymphocytes is the elimination of donor lymphocytes. However, unrestrained donor cell proliferation leading to TA-GVHD occurs when recipient lymphocytes are not functional or do not detect the donor lymphocytes as foreign. Preventing the donor lymphocytes from proliferating by exposing the lymphocytes in the blood products to various treatments should prevent or greatly diminish the incidence of TA-GVHD.

Abbreviations: CD8, cluster of differentiation 8; NK, natural killer; TA-GVHD, transfusion-associated graft-versus-host disease.
CD8+ and NK cells, would see the donor cells as self, and therefore not be expected to generate alloreactive responses toward the donor cells, while the donor cells would recognize the nonshared HLA haplotype on recipient cells as allogeneic and generate strong alloreactive responses. This particular donor/recipient combination has been observed to occur more frequently in populations that exhibit less heterogeneity, such as the Japanese (one in 874 unrelated transfusions) or when donor and recipient are related..$^{15,16}$ In Caucasians, it has been estimated that the homozygous donor/heterozygous recipient combination would occur at a frequency of one in 7,174. ${ }^{15,16}$ Because TA-GVHD does not occur with this frequency, it suggests that responses other than alloreactive CD8+ and NK cells are also important. Additional responses could include restricted cytotoxic $\mathrm{T}$ lymphocytes (CTL) recognizing minor histocompatibility antigens presented by the donor antigen-presenting cells..$^{17,18}$ In certain settings, dendritic cells have been shown to express granzyme B, which results in the inhibition/lysis of T cells that bind to these dendritic cells. ${ }^{9}, 19$

A second scenario conducive for the development of TA-GVHD occurs when recipient immune responses capable of eliminating donor cells are inhibited. There are several categories of individuals with suboptimal immune systems. Fetuses receiving intrauterine transfusion, premature infants, or neonates who require red blood cell (RBC) exchange are susceptible to TA-GVHD due to the immaturity of the neonatal immune system. Patients with hematopoietic malignancies and certain solid tumors or those receiving cancer treatments such as intensive chemotherapy, purine antimetabolites, or progenitor cell transplantation exhibit dysfunctional immune systems. Other individuals receiving granulocyte transfusions or those with genetic defects leading to congenital immunodeficiency are also at increased risk for TA-GVHD., ${ }^{2,20}$ The more complete the immunosuppression of the recipient's immune system, the more likely that TA-GVHD could result, even in fully allogeneic donor/recipient combinations.

\section{Methods for preventing TA-GVHD in at-risk patients}

The importance of the extensive proliferation of transfused donor T cells for the development of TA-GVHD and the inability to reverse the consequences of the donor antirecipient $T$ cell responses once initiated, caused investigators to focus on identifying treatments that could prevent donor $\mathrm{T}$ cell proliferation without interfering with the function of the transfused RBCs or platelets. Another concern was the introduction of new antigenic epitopes that could cause 
immune responses following transfusion of the treated blood products. One approach that was developed was the use of leukoreduction filters. The ability to deplete leukocytes (often more than five logs of WBCs) from the blood product did reduce the incidence of TA-GVHD. ${ }^{21,22}$ However, isolated cases of individuals who developed TA-GVHD when a limited number of leukocytes were transfused following leukoreduction have been reported. ${ }^{23}$ Thus, while there may be many benefits to the use of leukoreduction, it does not necessarily prevent the development of TA-GVHD.

Another approach that was tested was to expose the blood product to irradiation. An in vitro limiting dilution assay (LDA) was used to measure the effect of irradiation on $\mathrm{T}$ cell proliferation. ${ }^{24-26}$ The results of these in vitro studies led to the adoption of a dose of 25-30 Gy $\gamma$-irradiation as a standard for the inactivation of T lymphocytes in blood products. $^{2}$ This led to routine irradiation of blood products, especially in settings in which patients were considered to be at risk for developing TA-GVHD. In Japan, with the general population at greater risk for TA-GVHD because of less population diversity at the HLA complex, irradiation is uniformly applied to all transfusions. Recent summaries indicate that no further cases of TA-GVHD were detected once universal irradiation was implemented. ${ }^{27,28}$

\section{Technical advances}

While exposure of blood products to $\gamma$-irradiation is the standard of care, several drawbacks to this approach have been detected. Irradiated RBCs exhibit a decreased shelf life resulting from increased membrane permeability. This permeability leads to hemolysis and potassium leakage. These defects are improved when leukoreduction is used prior to irradiation. ${ }^{29-31}$ While $\gamma$-irradiation was found to inhibit TA-GVHD, it was much less effective at preventing other types of immune responses. ${ }^{32,33}$ WBCs that had been $\gamma$-irradiated were able to act as antigen- presenting cells and could therefore induce recipient immune responses. ${ }^{32-34}$ As a result, use of irradiated blood products did not reduce the incidence of alloimmunization in transfused individuals. ${ }^{35}$ In addition, the recent concern about the potential of using cesium found in the cesium source irradiators for bioterrorism has resulted in an increased regulatory burden, such as background checks for operators and restrictions on accessibility when using these irradiators. ${ }^{36}$ The use of X-rays instead of $\gamma$-irradiation is an alternative approach. A limited number of studies have compared X-rays to $\gamma$-irradiation and found that both approaches appear to be equally effective at interfering with lymphocyte proliferation. ${ }^{37-40}$

Pathogen reduction technologies were developed to reduce the incidence of pathogen transmission, both known and unknown, in blood products. The general approach for these protocols was to induce nucleic acid modifications in a manner that prevented replication of the pathogens (see Table 1 for a listing of the different pathogen reduction protocols). The ability of these protocols to interfere with replication by introducing nucleic acid modifications was also found to interfere with the proliferation of lymphocytes. This led to testing of the ability of different protocols to interfere with the function of $\mathrm{T}$ lymphocytes in different blood products. Comparison of the effectiveness of the pathogen reduction protocols with $\gamma$-irradiation using LDA found that these protocols were as effective as $\gamma$-irradiation in inhibiting $\mathrm{T}$ cell proliferation. ${ }^{32-34}$ Several different GVHD models were used to extend these in vitro results to in vivo settings. Initially, the parent into $F_{1}$ GVHD model, a murine model that replicates the donor/recipient combination that is most closely associated with TA-GVHD in humans, was tested using PEN110 and amotosalen plus ultraviolet (UV)-light-treated donor lymphocytes. The results indicated that treatment of the donor splenocytes with pathogen reduction protocols prevented development of GVHD in this model. ${ }^{41,42}$

Table I Pathogen reduction technologies

\begin{tabular}{|c|c|c|c|c|}
\hline Pathogen reduction agent & Company & Products treated & Current use & Reference(s) \\
\hline INACTINE (PENIIO) & VI Technologies & RBC & Halted & 51,52 \\
\hline Intercept & Cerus & Platelets & Used in Europe & 53,54 \\
\hline (S-59 + UV-light) & & Plasma & & \\
\hline S-303 & Cerus & RBC & In development & 55,56 \\
\hline \multirow[t]{3}{*}{ Mirasol (riboflavin + UV-light) } & TerumoBCT & Platelets & Used in Europe & 57 \\
\hline & & Plasma & Used in Europe & \\
\hline & & Whole blood & In development & \\
\hline Methylene blue + visible light or & MacoPharma & Plasma & Used in Europe & 58,59 \\
\hline UVC light & & Platelets & In development & \\
\hline Solvent detergent & Octapharma & Plasma & Used in Europe & 60,61 \\
\hline
\end{tabular}

Notes: VI Technologies, Watertown, MA, USA; Cerus, Concord, CA, USA; TerumoBCT, Lakewood, CO, USA; MacoPharma, Tourcoing, France; Octapharm, Lachen, Switzerland. Copyright (C) 2012 John Wiley and Sons. Reproduced with permission from Fast LD. Developments in the prevention of transfusion-associated graft-versus-host disease. Br J Haematol. 20I2; I58(5):563-568. ${ }^{2}$

Abbreviations: RBC, red blood cell; UV, ultraviolet; UVC, ultraviolet C. 
Xenogeneic GVHD models in which human peripheral blood mononuclear cells (PBMNC) infused into immunodeficient recipients attack the murine recipient have been used as a surrogate model for TA-GVHD. These models were used to test the in vivo responsiveness of treated human $\mathrm{T}$ lymphocytes. Xenogeneic GVHD results from human lymphocyte responses to antigens expressed by the recipient mice. Because normal mice mount strong responses to human cells, causing their elimination, xenogeneic GVHD has only been observed in immunodeficient mice, with the more immunodeficient the strain, the more potent the xenogeneic GVHD response. Initially, severe combined immunodeficient (SCID) mice lacking B and T cells were used as recipients to study xenogeneic GVHD responses. ${ }^{4-45}$ Because of the variable induction of xenogeneic GVHD in SCID mice despite the use of large numbers of donor PBMNC and administration of cytokines, investigators moved to utilizing Rag2-/- $\gamma_{c}-/-$ double knockout mice lacking B, T, and NK cells that had received $350 \mathrm{cGy}$ total body irradiation prior to intravenous injection; it was observed that the use of these recipients improved the frequency of xenogeneic GVHD while using fewer donor PBMNC. ${ }^{46}$ Several studies testing the effect of PEN110 treatment and the effect of riboflavin plus UV light on xenogeneic GVHD responses were conducted using these recipient mice. ${ }^{47,48}$ Studies conducted more recently have observed that using NODScidIL2r $\gamma^{\text {null }}$ knockout mice receiving $200 \mathrm{cGy}$ total body irradiation required fewer PBMNC to more rapidly and uniformly induce xenogeneic GVHD. ${ }^{49}$ Use of the NODScidIL2 $r \gamma^{\text {null }}$ knockout mice was found to result in more uniform results when testing the xenogeneic GVHD responses of riboflavin + UV-light-treated PBMNC.$^{33}$ The cumulative results of the studies with in vivo GVHD models supports the concept that pathogen reduction protocols could be used to prevent TA-GVHD as effectively as $\gamma$-irradiation.

One concern with the use of pathogen reduction protocols is that the treatment process introduces neoantigens that could trigger immune responses. This was observed for treatment of blood products with Pen 110 when the protocol was tested in clinical trials. It was observed that when Pen110-treated blood products were transfused into sickle cell patients, they uniformly developed antibodies to the treated cells by the second transfusion. Antibodies were also generated in surgical patients who had received treated blood products, especially after the patients developed an infection. Transfusion of S-303-treated RBCs was also demonstrated to be capable of inducing antibodies in a subset of patients. ${ }^{50}$

\section{Future perspectives}

The use of pathogen reduction protocols for the treatment of blood products is being used extensively outside of the US. There have been no reports of TA-GVHD occurring following transfusion of these treated products. Cost and efficiency considerations would indicate that the ability to treat whole blood using the pathogen reduction protocols prior to separation into the different components would be advantageous. Treatment of whole blood with pathogen reduction protocols is ongoing, and initial studies have shown that conditions can be identified that are capable of inhibiting $\mathrm{T}$ lymphocyte proliferation in whole blood as effectively as $\gamma$-irradiation. ${ }^{33}$ This could provide a method to efficiently and effectively treat blood to prevent TA-GVHD. While the use of treated whole blood or products derived from treated whole blood is being moved to clinical studies, the results of these studies are needed before this technology can be licensed and used routinely.

\section{Conclusion}

TA-GVHD occurs when there is unrestrained proliferation of transfused donor lymphocytes. This occurs when the recipient's ability to mediate elimination of the donor cells following transfusion is compromised either because of the lack of genetic disparity between the donor and recipient or because the recipient's immune system is suppressed. Bone marrow aplasia induced by donor anti-recipient responses makes it difficult to reverse the course of TA-GVHD once it has begun. Thus, the focus for the prevention of TA-GVHD has been to prevent donor $\mathrm{T}$ cell proliferation. Currently, leukoreduction and/or $\gamma$-irradiation are used to prevent TA-GVHD. Despite the effectiveness of $\gamma$-irradiation in preventing TA-GVHD, there are drawbacks to the use of $\gamma$-irradiation, including regulatory concerns about the use of irradiators and an inability to prevent recipient anti-donor immune responses. A potentially more effective alternative to $\gamma$-irradiation is the use of blood products treated with pathogen reduction protocols, as a number of studies have demonstrated that pathogen reduction protocols are as effective as $\gamma$-irradiation at preventing $\mathrm{T}$ cell responses but do not have as many drawbacks and would provide an alternative method for overcoming the increasing number of constraints placed on the use of cesium source irradiators. While the use of pathogen reduction protocols provides an alternative approach for preventing TA-GVHD, it has not been approved for use in all countries. Ongoing studies will provide a basis for moving forward with the implementation of pathogen reduction protocols. 


\section{Disclosure}

The author has received grant funding from VI Technologies and from TerumoBCT Technologies (formerly CaridianBCT Technologies and NavigantBCT Technologies). The author reports no other conflict of interest in this work.

\section{References}

1. Dwyre DM, Holland PV. Transfusion-associated graft-versus-host disease. Vox Sang. 2008;95(2):85-93.

2. Fast LD. Developments in the prevention of transfusion-associated graft-versus-host disease. Br J Haematol. 2012;158(5):563-568.

3. Lee TH, Donegan E, Slichter S, Busch MP. Transient increase in circulating donor leukocytes after allogeneic transfusions in immunocompetent recipients compatible with donor cell proliferation. Blood. 1995;85(5):1207-1214.

4. Fast LD. Recipient CD8+ cells are responsible for the rapid elimination of allogeneic donor lymphoid cells. J Immunol. 1996;157(11): 4805-4810.

5. Fast LD. Recipient elimination of allogeneic lymphoid cells: donor CD4(+) cells are effective alloantigen-presenting cells. Blood. 2000;96(3):1144-1149.

6. Waggoner SN, Cornberg M, Selin LK, Welsh RM. Natural killer cells act as rheostats modulating antiviral T cells. Nature. 2012;481(7381): 394-398.

7. Welsh RM, Waggoner SN. NK cells controlling virus-specific T cells: rheostats for acute vs persistent infections. Virology. 2013;435(1): $37-45$.

8. Jahrsdörfer B, Vollmer A, Blackwell SE, et al. Granzyme B produced by human plasmacytoid dendritic cells suppresses T-cell expansion. Blood. 2010;115(6):1156-1165.

9. Karrich JJ, Jachimowski LC, Nagasawa M, et al. IL-21-stimulated human plasmacytoid dendritic cells secrete granzyme B, which impairs their capacity to induce T-cell proliferation. Blood. 2013;121(16): 3103-3111.

10. Sivori S, Carlomagno S, Falco M, Romeo E, Moretta L, Moretta A Natural killer cells expressing the KIR2DS1-activating receptor efficiently kill T-cell blasts and dendritic cells: implications in haploidentical HSCT. Blood. 2011;117(16):4284-4292.

11. Hu B, He Y, Wu Y, et al. Activated allogeneic NK cells as suppressors of alloreactive responses. Biol Blood Marrow Transplant. 2010;16(6): $772-781$.

12. Olson JA, Leveson-Gower DB, Gill S, Baker J, Beilhack A, Negrin RS. NK cells mediate reduction of GVHD by inhibiting activated, alloreactive T cells while retaining GVT effects. Blood. 2010;115(21): 4293-4301.

13. Reagan JL, Fast LD, Safran H, et al. Cellular immunotherapy for refractory hematological malignancies. J Transl Med. 2013;11:150.

14. Otsuka S, Kunieda K, Kitamura F, et al. The critical role of blood from HLA-homozygous donors in fatal transfusion-associated graft-versus-host disease in immunocompetent patients. Transfusion. 1991;31(3):260-264.

15. Ohto H,Anderson KC. Survey of transfusion-associated graft-versus-host disease in immunocompetent recipients. Transfus Med Rev. 1996;10(1): 31-43.

16. Ohto H, Yasuda H, Noguchi M, Abe R. Risk of transfusion-associated graft-versus-host disease as a result of directed donations from relatives. Transfusion. 1992;32(7):691-693.

17. Goulmy E. Minor histocompatibility antigens: from transplantation problems to therapy of cancer. Hum Immunol. 2006;67(6):433-438.

18. Spellman S, Warden MB, Haagenson M, et al. Effects of mismatching for minor histocompatibility antigens on clinical outcomes in HLAmatched, unrelated hematopoietic stem cell transplants. Biol Blood Marrow Transplant. 2009;15(7):856-863.
19. Zangi L, Klionsky YZ, Yarimi L, et al. Deletion of cognate CD8 T cells by immature dendritic cells: a novel role for perforin, granzyme A, TREM-1, and TLR7. Blood. 2012;120(8):1647-1657.

20. King KE, Ness PM. How do we prevent transfusion-associated graftversus-host disease in children? Transfusion. 2011;51(5):916-920.

21. Hayashi H, Nishiuchi T, Tamura H, Takeda K. Transfusion-associated graft-versus-host disease caused by leukocyte-filtered stored blood. Anesthesiology. 1993;79(6):1419-1421.

22. Stainsby D, Jones H, Asher D, et al; SHOT Steering Group. Serious hazards of transfusion: a decade of hemovigilance in the UK. Transfus Med Rev. 2006;20(4):273-282.

23. Akahoshi M, Takanashi M, Masuda M, et al. A case of transfusion-associated graft-versus-host disease not prevented by white cell-reduction filters. Transfusion. 1992;32(2):169-172.

24. Luban NL. Prevention of transfusion-associated graft-versus-host disease by inactivation of T cells in platelet components. Semin Hematol. 2001;38(4 Suppl 11):34-45.

25. Luban NL, Drothler D, Moroff G, Quinones R. Irradiation of platelet components: inhibition of lymphocyte proliferation assessed by limiting-dilution analysis. Transfusion. 2000;40(3):348-352.

26. Pelszynski MM, Moroff G, Luban NL, Taylor BJ, Quinones RR. Effect of gamma irradiation of red blood cell units on T-cell inactivation as assessed by limiting dilution analysis: implications for preventing transfusion-associated graft-versus-host disease. Blood. 1994;83(6): 1683-1689.

27. Uchida S, Tadokoro K, Takahashi M, Yahagi H, Satake M, Juji T. Analysis of 66 patients definitive with transfusion-associated graft-versus-host disease and the effect of universal irradiation of blood. Transfus Med. 2013;23(6):416-422.

28. Otsubo H, Yamaguchi K. Current risks in blood transfusion in Japan. Jpn J Infect Dis. 2008;61(6):427-433.

29. Harm SK, Raval JS, Cramer J, Waters JH, Yazer MH. Haemolysis and sublethal injury of RBCs after routine blood bank manipulations. Transfus Med. 2012;22(3):181-185.

30. Hirayama J, Abe H, Azuma H, Ikeda H. Leakage of potassium from red blood cells following gamma ray irradiation in the presence of dipyridamole, trolox, human plasma or mannitol. Biol Pharm Bull. 2005;28(7):1318-1320.

31. Ran Q, Hao P, Xiao Y, Zhao J, Ye X, Li Z. Effect of irradiation and/ or leucocyte filtration on RBC storage lesions. PLoS One. 2011;6(3): e18328.

32. Fast LD, Dileone G, Li J, Goodrich R. Functional inactivation of white blood cells by Mirasol treatment. Transfusion. 2006;46(4):642-648.

33. Fast LD, Nevola M, Tavares J, Reddy HL, Goodrich RP, Marschner S. Treatment of whole blood with riboflavin plus ultraviolet light, an alternative to gamma irradiation in the prevention of transfusion-associated graft-versus-host disease? Transfusion. 2013;53(2):373-381.

34. Fast LD, DiLeone G, Marschner S. Inactivation of human white blood cells in platelet products after pathogen reduction technology treatment in comparison to gamma irradiation. Transfusion. 2011;51(7): 1397-1404.

35. Ohto H. Gamma radiation does not prevent transfusion-induced HLA alloimmunization. Transfusion. 1997;37(8):878-879.

36. Mintz PD. Cesium cessation? An advantage of pathogen reduction treatments. Transfusion. 2011;51(7):1369-1376.

37. Herva E, Kiviniity K. The effect of in vitro irradiation on the responses of human lymphocytes to PHA, PPD and allogeneic cells. Strahlentherapie. 1975;149(5):504-512.

38. Janatpour K, Denning L, Nelson K, Betlach B, Mackenzie M, Holland P. Comparison of X-ray vs gamma irradiation of CPDA-1 red cells. Vox Sang. 2005;89(4):215-219.

39. Klein HG. Transfusion-associated graft-versus-host disease: less fresh blood and more gray (Gy) for an aging population. Transfusion. 2006;46(6):878-880.

40. Moroff G, Luban NL. The irradiation of blood and blood components to prevent graft-versus-host disease: technical issues and guidelines. Transfus Med Rev. 1997;11(1):15-26. 
41. Fast LD, DiLeone G, Edson CM, Purmal A. Inhibition of murine GVHD by PEN110 treatment. Transfusion. 2002;42(10):1326-1332.

42. Grass JA, Wafa T, Reames A, et al. Prevention of transfusionassociated graft-versus-host disease by photochemical treatment. Blood. 1999;93(9):3140-3147.

43. Hoffmann-Fezer G, Gall C, Zengerle U, Kranz B, Thierfelder S. Immunohistology and immunocytology of human T-cell chimerism and graft-versus-host disease in SCID mice. Blood. 1993;81(12):3440-3448.

44. Roychowdhury S, Blaser BW, Freud AG, et al. IL-15 but not IL-2 rapidly induces lethal xenogeneic graft-versus-host disease. Blood. 2005;106(7):2433-2435.

45. Sandhu JS, Gorczynski R, Shpitz B, Gallinger S, Nguyen HP, Hozumi N. A human model of xenogeneic graft-versus-host disease in SCID mice engrafted with human peripheral blood lymphocytes. Transplantation. 1995;60(2):179-184.

46. van Rijn RS, Simonetti ER, Hagenbeek A, et al. A new xenograft model for graft-versus-host disease by intravenous transfer of human peripheral blood mononuclear cells in RAG2-/- gamma c-/- double-mutant mice. Blood. 2003;102(7):2522-2531.

47. Fast LD, DiLeone G, Cardarelli G, Li J, Goodrich R. Mirasol PRT treatment of donor white blood cells prevents the development of xenogeneic graft-versus-host disease in Rag2-/-gamma c-/- double knockout mice. Transfusion. 2006;46(9):1553-1560.

48. Fast LD, Semple JW, DiLeone G, et al. Inhibition of xenogeneic GVHD by PEN110 treatment of donor human PBMNCs. Transfusion. 2004;44(2):282-285.

49. King MA, Covassin L, Brehm MA, et al. Human peripheral blood leucocyte non-obese diabetic-severe combined immunodeficiency interleukin-2 receptor gamma chain gene mouse model of xenogeneic graft-versus-host-like disease and the role of host major histocompatibility complex. Clin Exp Immunol. 2009;157(1):104-118.

50. Benjamin RJ, McCullough J, Mintz PD, et al. Therapeutic efficacy and safety of red blood cells treated with a chemical process (S-303) for pathogen inactivation: a Phase III clinical trial in cardiac surgery patients. Transfusion. 2005;45(11):1739-1749.
51. AuBuchon JP, Pickard CA, Herschel LH, et al. Production of pathogeninactivated RBC concentrates using PEN110 chemistry: a Phase I clinical study. Transfusion. 2002;42(2):146-152.

52. Purmal A, Valeri CR, Dzik W, et al. Process for the preparation of pathogen-inactivated RBC concentrates by using PEN110 chemistry: preclinical studies. Transfusion. 2002;42(2):139-145.

53. Pineda A, McCullough J, Benjamin RJ, et al; SPRINT Study Group. Pathogen inactivation of platelets with a photochemical treatment with amotosalen $\mathrm{HCl}$ and ultraviolet light: process used in the SPRINT trial. Transfusion. 2006;46(4):562-571.

54. Singh Y, Sawyer LS, Pinkoski LS, et al. Photochemical treatment of plasma with amotosalen and long-wavelength ultraviolet light inactivates pathogens while retaining coagulation function. Transfusion. 2006;46(7):1168-1177.

55. North A, Ciaravino V, Mufti N, Corash L. Preclinical pharmacokinetic and toxicology assessment of red blood cells prepared with S-303 pathogen inactivation treatment. Transfusion. 2011;51(10): 2208-2218.

56. Winter KM, Johnson L, Kwok M, et al. Red blood cell in vitro quality and function is maintained after $\mathrm{S}-303$ pathogen inactivation treatment. Transfusion. 2014;54(7):1798-1807.

57. Marschner S, Goodrich R. Pathogen reduction technology treatment of platelets, plasma and whole blood using riboflavin and UV light. Transfus Med Hemother. 2011;38(1):8-18.

58. Lozano M, Cid J, Müller TH. Plasma treated with methylene blue and light: clinical efficacy and safety profile. Transfus Med Rev. 2013;27(4): 235-240.

59. Seghatchian J, Tolksdorf F. Characteristics of the THERAFLEX UV-Platelets pathogen inactivation system - an update. Transfus Apher Sci. 2012;46(2):221-229.

60. Hellstern P, Solheim BG. The use of solvent/detergent treatment in pathogen reduction of plasma. Transfus Med Hemother. 2011;38(1):65-70.

61. Liumbruno GM, Franchini M. Solvent/detergent plasma: pharmaceutical characteristics and clinical experience. J Thromb Thrombol. Epub May 21, 2014.
International Journal of Clinical Transfusion Medicine

\section{Publish your work in this journal}

International Journal of Clinical Transfusion Medicine is an international, peer-reviewed, open access, online journal publishing clinicalexperimental, policy-making and evidence-based practices of all topics pertaining to clinical transfusion medicine. Original research, short reports, reviews, case reports and commentaries are invited.

\section{Dovepress}

The manuscript management system is completely online and includes a very quick and fair peer-review system, which is all easy to use. Visit http://www.dovepress.com/testimonials.php to read real quotes from published authors. 